\title{
Distance Education and Distributed Virtual Environments
}

H. Trefftz, C. Correa, M. A. González, G. Imbeau, J. Restrepo, M. I. Vélez and C. Trefftz

Universidad EAFIT

A.A. 3300

Medellín

Colombia-SouthAmerica

e-mail:htrefft@@sigma.eafit.edu.co .

Funding for this project has been provided by EAFIT University and Colciencias-BID under grant number 150-97.

\begin{abstract}
The possible applications of Distributed Virtual Environments to support Distance Education are being explored. A first experience with IRC (Internet Relay Chat) is described.
\end{abstract}

Distance education, Virtual reality

\section{Keywords}




\section{INTRODUCTION}

Distributed Virtual Environments (Burdea 1994, Broll 1995, Benford 1995, Hagsand 1996, Wexelblat 1993) are benefiting from two trends in the computer and communication marketplace: The increase in speed of networks and microprocessors and the decrease in price of hardware. These trends open the door to a number of new applications, among others "Virtual Classrooms" (O'Malley 1995, Kelsel 1996, Pantelidis 1994).

The project described in this paper explores the possible applications of Distributed Virtual Environments in the area of distance education. Researchers working on the project include both educators and computer scientists. The project is planned to be developed in a number of stages. In the first stage, the concept of IRC (Internet Relay Chat) is being used as a basis for a first interface that will allow an instructor and a number of students to interact in "real" time. In a second phase of the project, 3D "immersive" environment will be incorporated in the interface.

In this paper the results of a first implementation of a modified version of IRC, called AVALON, the critique by the educators in the group of this first version and the resulting new version of AVALON are described. We also describe the long term direction of the project.

The rest of this paper is structured as follows. Section 2 describes the preliminary version of AVALON.

The critique to the first interface by the educators in our team is included in section 3. Section 4 contains the modifications performed on the first version of the software. Section 5 describes the experiments that will be performed next semester. Conclusions and future work can be found in section 6.

\section{INITIAL VERSION OF AVALON}

The structure of IRC is based upon 2 assumptions:

- All users are equal.

- Users are logged on to machines that are not necessarily in the same domain.

In the kind of environment being envisioned for this project, these assumptions do not hold.

The interaction among the participants will be directed by the instructor. A student who wishes to participate should ask the instructor before starting to communicate to the rest of the group. Therefore in AVALON, there are two different user interfaces available: One for the instructor, and one for students.

The instructor receives the requests for participation from the students and "yields the floor" to a given student. In this first version of AVALON, a student wishing to speak signals the instructor by clicking on a particular icon which results in a square associated with the student flashing on the screen of the instructor and a 
message appearing in the common text area telling everybody that the given student wishes to talk.

It is also assumed that the students and the instructor will be connected through a dial-in service provided by an academic institution. Thus all users will be in the same segment. Therefore the implementation uses multicast services to improve the response time. As multicast services become more widely available through Internet, using multicast will be an alternative that will improve performance.

\section{CRITIQUE OF AVALON'S FIRST VERSION}

The members in our team with a formal background in education had the following critique about the first version of AVALON: Distance Education is based upon the assumption that the student does NOT have to be at a certain time in a given place. This system will require the student to have access to a personal computer with the appropriate connection. The concept of physical distance is kept but the participants have to be "present" at the same time.

The number of simultaneous participants cannot currently exceed three or four persons. Beyond four users, the interactions become so fast that it is hard to read what the other users are saying/writing. The fact that the system is appropriate for a small number of participants only eliminates a comparative advantage of distance education: It covers a large and sparse population that cannot attend, for reasons of time and space, a regular educational institution. Furthermore Distance Education is an affordable alternative for this population. Limiting the size of the groups has an effect in costs as it will require a larger number of facilities.

Virtual Environments would become an enabling technology: They will enable students to participate, to interact with an instructor and with other students. On the other hand it would diminish the opportunity of advancing at one's own self pace in one's available spare time.

This discussion has lead the team to refocus the goals of the project. The goal will not be to replace the classroom and to expect all students to be "present" in the virtual classroom at once. Rather, the tool will be a "virtual help-room" where the instructor will be present at certain times and the students can "come" when the instructor arranges a meeting. The traditional distance educational media will not be replaced by the virtual help-room. The virtual help-room will be an additional service available. It will enable students to interact with an instructor and with each other in ways that were not previously available in Distance Learning.

\section{AN IMPROVED VERSION OF AVALON}

The following changes were made to AVALON:

- To avoid a particular student annoying the instructor and the rest of the students with excessive requests for participation, a request for an opportunity to speak will 
now result on the change of an icon that represents the student but no text will appear on the common text area. Thus, the instructor will realize that a particular student wants to participate in the discussion by looking at the corresponding icon. Any further requests will not cause any additional effects.

- The observation that no more than 4 students should be active at once lead to a redesign of the interface for the instructor. Every active student in a session will have a small face icon associated. The shape of the face reflects the current status of the student. The possible states are:

1. Listening and understanding (happy face)

2. Listening and not understanding (sad face)

3. Requesting the chance to speak

4. Requesting the chance to send a private message to the instructor

5. Absent but will return soon

6. Absent and will not return

\section{GENERAL OVERVIEW OF THE PROJECT}

The project started this year. The project has two main stages. The first one will use a text based interface. In the second one, we will develop an immersive virtual environment including sound, 3D avatars and other media.

The experiment will be performed three times:

1. Once with the text-based version of the interface.

2. Once with the graphic interface with one-to-one interaction between the instructor ond one student.

3. Once with the graphic interface with many-to-many interaction between the instructor and a group of students.

\subsection{The first experiment}

The first experiment started in August of 1997 using the text-based version of the interface. An introductory course on Environmental Sciences is being taught to eight first-year college students. The group was divided into two subgroups: One is receiving the course using traditional Distance Education methods and the other one is using the text-based interface. A tutor was assigned to each group. The tutors are in charge of guiding the modules of the course being taught. A comparison will be performed between both groups. We present the results obtained in this first experience under two categories: Challenges and Strategies. The Challenges are the situations on which we must continue working to make the experience more effective and the Strategies are the tools or strengths that we relied on during this first experience. 


\section{Challenges}

The instructor needs to acquire previous experience in using the interface and must be very familiar with the entire course, so he/she has an appropriate knowledge of the situations and problems that could arise during the use of the tool. The instructor and students must concentrate on the interaction; the interface should be as "transparent" as possible. It is also necessary to be more familiar with the procedures that the students are following when using the tool.

The tutor needs to have a plan for the class and he/she must be familiar with the key concepts in the modules.

Adequate pedagogical resources are necessary to make the tutorial sessions more dynamic. For instance: If a student has difficulties and is slowing down the pace of the group with a repetitive question, the tutor should ask him/her to find the answer to his/her question on the written material.

The students should study deeply the modules of the course and if possible they should prepare some questions before every session.

The tutor has to be able to maintain several conversations at once when using the tool.

Although, the tool offers the students the opportunity of telling the instructor how are they feeling in the session, the tutor misses the non-verbal clues that students provide in a regular classroom environment.

\section{Strategies}

Having a small number of students in every session (four).

The ability given to the tutor of yielding the floor to a student and being able to take the floor back has made the interaction much more orderly and more dynamic.

Both the students and the tutor have found the possibility given to the students to express their mood very useful. The tutor can react according to the feedback provided by the students.

We also want to emphasize several technical aspects of the future stages of the project:

Immersive Interface: In the immersive interface the participants will find themselves in a Virtual Classroom. Each student and the instructor will have a 3D simplified representation, called an Avatar.

The avatar will be the means of the participants to share non-verbal information with the other participants. Via the avatar, the user will be able to:

- Occupy a space

- Move inside the world

- See the location and movements of the other participants

- Ask for the right to speak

The interface will also handle voice. According to a certain protocol, the participants will be able to hear others and to speak to others. Based on the current experience with the text interface, we are planning to have only the instructor talking to the group by default. If a student wants to speak to the group, he/she will "raise their virtual hands" asking the teacher for the opportunity to speak. 
Only the instructor can "yield" the ability to talk to the group to a student (and take it back) whenever he/she wants.

The individual workstations will have a digitizing pad. According to a similar protocol, the participants will have access to a White Board. When a participant writes on the white board, whatever he/she writes on the digitizing pad on his/her workstation, appears magnified on the virtual white board. This offers a means of sharing diagrams with the group.

Other media have been proposed, for instance a VCR and a cassette player, but this media takes a lot of bandwidth (communications speed). Experiments will be conducted to determine the viability of such media on different communication networks.

On the long run we are also considering importing Virtual Worlds (described with the VRML language Brol 1995) to our system. This would allow the group to take a "Virtual Field trip" to virtually anywhere.

\subsection{Computer Networks}

The system will be tried on two types of computer networks:

-On local area networks (LANs), with high bandwidth,

-On wide area networks (WANs), where the communication bandwidth is much lower.

There are some interesting challenges when using WANs. Some compromises will have to be made in order to maintain a reasonable response time. No part of a voice message, for instance, can be dropped, whereas the message describing the movement of an avatar inside the world can be delayed.

\section{CONCLUSIONS AND FUTURE WORK}

Our vision is having our system used as support for distance learning in our country. Our hypothesis is that the use of such a system would improve the quality of distance learning.

The presence of both educators and computer scientists in our group has resulted in discussions and improvements to the prototypes we are developing.

IRC was modified to provide a more orderly and richer in information environment for class interaction. Our preliminary tests seem to indicate that only a small number of users can interact effectively, from an educational point of view. 


\section{REFERENCES}

Benford (1995),"User embodiment in Collaborative Environments ",S Benford and $\mathrm{J}$ Bowers and L Fahlen and C Greenhalgh and D Snowdown, Proceedings of CHI 95, Denver ,Colorado.

Broll (1995), "Adding Multi-User Support to VRML, W Broll and D England, Proceedings of the VRMUL 95 Symposium ACM,pp 87-94, Denver, Colorado,

Burdea (1994), "Virtual Reality Technology", Grigore Burdea, John Willey and Sons.

O'Malley (1995), "Computer Supported Collaborative Learning", C O'Malley, Springer Verlag.

Wexelblat (1993), "The Reality of Cooperation: Virtual Reality and CSCW" in "Virtual Reality: Applications and Explorations", A Wexelblat, Academic Publishers, Academic Publishers.

Hagsand (1996), "Interactive Multiuser VEs in the DIVE System", Olaf Hagsand,IEEE Multimedia, Spring.

Kelsel (1996), VR in Education, Sandra Helsel, VR News, July.

Pantelidis (1994), "Exploring Virtual Reality for Classroom Use", V Pantelidis and L Auld, Tech Trends, Jan/Feb.

IVU (1996), Virutal University's Home Page, http://www.vu.org/.

\section{BIOGRAPHIES}

Helmuth Trefftz. Currently studying a Ph.D. at Rutgers Unviersity. Interests: Distributed Multiuser Virtual Reality.

Carlos David Correa. Finishing undergraduate studies at EAFIT University. Interests: Computer Graphics for Virtual Reality.

Miguel Angel Gonzalez. Invited scholar at EAFIT University.

Interests: Evaluation of Education processes.

Gilles Imbeau, Ph.D. Professor at Universite de Quebec au Chicoutimic. Interests: Computer Supported Learning.

Juliana Restrepo. Invited researcher at EAFIT University. Pre School Teacher at Gimnasio Los Pinares. Interests: Computer Supported Collaboravie Learning

Maria Isabel Velez. Gradute student in Paleontholgy, Scotland. Interests: Education and Earth Sciences.

Christian Trefftz, Ph.D. Professor at EAFIT University. Interests: Parallel computing, distributed systems, Distributed Virtual Reality. 